EESTI NSV TEADUSTE AKADEEMIA TOIMETISED 1952. I k, nr. 1 ИЗВЕСТИЯ АКАДЕМИИ НАУК ЭСТОНСКОИ ССР 1952. ТоМ I, 리 1

\title{
О ГИПЕРБОЛИЧЕСКОЙ МЕХАНИКЕ И ВОПРОСАХ КОСМОГОНИИ
}

\author{
Ю. Ю. НУТ, \\ действительный член Академии Наук Эстонской ССР
}

\section{1. Инерциальная система}

В последующем излагаются некоторые положения, определяющие разновидность механики, названной «гиперболической», и дающей новый подход к некоторым вопросам космогонии.

Предполагается строго евклидовое трехмерное пространство, в котором каким-либо способом зафиксирована прямоугольная система координат, начало $O$ которой неизменно связано с материальной точкой, где физически задан также отсчет времени $t$. Положение любой материальной точки $P$ в момент $t$ тогда определено координатами $x_{\alpha}, \alpha=1,2,3$.

Вводится постоянная $\sigma$, получающаяся из красного смещения опектров внегалактических туманностей, а именно,

$$
\sigma=1,6 \cdot 10^{-17}\left[\text { сек }^{-1}\right] \text {. }
$$

Применяя для обозначения производных по времени точечную символику, условимся вектор $x_{\alpha}-\sigma x_{\alpha}$ называть «собственной» скоростью материальной точки $P$. Вводя для этой собственной скорости символ $v$, имеем следовательно:

$$
\dot{x}_{\alpha}=v_{\alpha}+\sigma x_{\alpha} .
$$

Абсолютную величину вектора $v_{\alpha}$ будем обозначать символом $v$.

Вектор $\sigma x_{\alpha}$ условимся называть «инерциальной скоростью» точки $P$. Если ввести еще вектор $q_{\alpha}$ посредством

$$
q_{\alpha}=x_{\alpha} e^{-\sigma t}
$$

то получается

$$
v_{\alpha}=\dot{q}_{\alpha} e^{\sigma t}
$$

Числа $q_{\alpha}$ будем называть «приведенными» координатами точки $P$.

Предположим, что систему координат удалось подобрать так, что величины собственных скоростей оказываются ограниченными. Предположим, что верхним пределом собственной скорости является с $=3 \cdot 10^{10}$ [см сек $\left.{ }^{-1}\right]$, т. е. собственная скорость фотона. В указанной системе координат собственная скорость материальной частицы, имеющей неисчезающую массу покоя, должна быть тогда меньше $c$. 
Исходя именно из такой системы координат, условимся понимать под «инерциальной системой $O$ » совокупность всех тех материальных точек, у которых собственная скорость $v$ равна нулю. Согласно (1) такие ма. териальные точки все же расходятся. Предположим, что для всех материальных точек инерциальной системы $O$ время $t$ отсчитывается одинаково.

Перенесем начало координат из $O$ в другую материальную точку $P$ той же инерциальной системы, закрепив новое начало теперь уже в $P$. Для простоты допустим, что новые оси параллельны старым. Рассмотрим произвольно избранную третью материальную точку $Q$, могущую и не принадлежать к исходной инерциальной системе $O$. Пусть $Q$ имеет в фиксированный момент времени $t$ исходной инерциальной системы при начале координат $O$ координаты $x^{\prime} \alpha$, а при начале $P-$ координаты $x^{\prime \prime} \alpha$. Пусть притом точка $P$ при начале $O$ имеет координаты $x_{\alpha}$. Тогда

$$
x_{\alpha}^{\prime \prime}=x_{\alpha}^{\prime}-x_{\alpha},
$$

откуда, в силу одинаковости времени $t$ в пределах инерциальной системы,

$$
\dot{x}_{\alpha}^{\prime \prime}=\dot{x}_{\alpha}^{\prime}-\dot{x}_{\alpha}=\dot{x}_{\alpha}^{\prime}-\sigma x_{\alpha}=\dot{x}_{\alpha}^{\prime}-\sigma x_{\alpha}^{\prime}+\sigma x_{\alpha}^{\prime \prime},
$$

T. e.

$$
\dot{x}_{\alpha}^{\prime \prime}-\sigma x_{\alpha}^{\prime \prime}=\dot{x}_{\alpha}^{\prime}-\sigma x_{\alpha}^{\prime} .
$$

Это значит, что собственная скорость точки $Q$ не зависит от выбора начала координат в инерциальной системе. Таким образом, собственная скорость любой материальной точки $Q$ может рассматриваться как собственная скорость по отношению ко всей инерциальной системе.

\section{2. Импульс и энергия}

При построении гиперболической механики в основу кладется принцип, согласно которому импульс и энергия зависят от собственной скорости, но не зависят от скорости инерциальной.

Импульс ( количество движения) $p_{\alpha}$ представляет вектор

$$
p_{\alpha}=M v_{\alpha}
$$

где $M$ масса частицы при собственной скорости $v_{\alpha}$.

Если в пространстве действует скалярный потенциал II, то в соответствии с обычными концепциями и в гиперболической механике постулируется, что

$$
\dot{p}_{\alpha}=-\frac{\partial \Pi}{\partial x_{\alpha}}
$$

Далее, однако, получаются две разновидности гиперболической динамики.

1) Вариант $\mathrm{N}$ (Ньютон): $M$ не зависит от $v_{\alpha}$ и $t$; тогда, положив $M=m$, имеем

$$
\dot{p}_{\alpha}=m \dot{v}_{\alpha}
$$

Под собственной энергией частицы в этом варианте подразумевается скаляр $E$, где

$$
E=\frac{1}{2} m v^{2}, \quad v^{2}=v_{\alpha} v_{\alpha}=v_{1}^{2}+v_{9}^{2}+\tau_{3}^{2}
$$

(повторение индекса включает требование суммирования соответствующих слагаемых по всем значениям индекса). 
Путем дифференцирования по $t$ отсюда сразу же получается

$$
\dot{E}=\dot{p}_{\alpha} v_{\alpha} \text {. }
$$

2) Вариант $E$ (Эйнштейн): $M$ зависит от $v$ по закону

$$
M=\frac{m}{\sqrt{1-\frac{v^{2}}{c^{2}}}},
$$

где $m$ опять-таки постоянная (масса покоя), а $c$ собственная скорость света в пустоте.

Вводя вспомогательную переменную ш посредством

$$
v=c \text { th }(1)
$$

имеем тогда

$$
M=m \mathrm{ch} \omega .
$$

Под собственной энергией в варианте $\mathrm{E}$ понимаем скаляр $E$, определяемый как

$$
E=M c^{2}=m c^{2} \text { ch } \omega .
$$

При $v=0$, т. е. $\omega=0, E$ превращается в собственную энергию покоя частицы, равную

$$
E_{\circ}=m c^{2} \text {. }
$$

Положим еще

$$
v_{\alpha}=c \text { th } \omega_{\alpha},
$$

причем, очевидно,

$$
\text { th }^{2} \omega_{1}+\text { th }^{2} \omega_{2}+\text { th }^{2} \omega_{3}=t^{2}{ }^{2} \omega .
$$

Тогда импульс $p_{\alpha}$ принимает вид

$$
p_{\alpha}=m c \text { ch } \omega \text { th } \omega_{\alpha} .
$$

Из (6), (7) и (8) легко выводится, на основании свойств гиперболических функций,

$$
c^{2} p_{\alpha} p_{\alpha}+E_{\circ}^{2}=E^{2} .
$$

Дифференцируя это по времени $t$, получаем:

$$
\dot{E}=c^{2} \dot{p_{\alpha}} p_{\alpha}
$$

T. e.

$$
\dot{E}=c^{2} \frac{\dot{p}_{\alpha} p_{\alpha}}{E}=c^{2} \frac{\dot{p}_{\alpha} M v_{\alpha}}{M c^{2}}=\dot{p}_{\alpha} v_{\alpha}
$$
ники.

Стало быть, соотношение (4) справедливо в обоих вариантах меха-

\section{3. Консервативные потенциалы}

Для определенных категорий потенциала II закон сохранения полной энергии соблюдается и в гиперболической механике. Такие потенциалы П можно назвать консервативными.

1) ПІ зависит только от приведенных координат $q_{\alpha}$, т. е.

$$
\Pi \equiv \Pi\left(q_{1}, q_{2}, q_{3}\right) .
$$


Тогда

$$
\begin{gathered}
\mathrm{II}=\frac{\partial \mathrm{II}}{\partial t}+\frac{\partial \Pi}{\partial x_{\alpha}} \dot{x}_{\alpha}=\frac{\partial \Pi}{\partial t}-\dot{p}_{\alpha} x_{\alpha}=\frac{\partial \Pi}{\partial t}-\dot{p}_{\alpha}\left(v_{\alpha}+\sigma x_{\alpha}\right)= \\
=\frac{\partial \Pi}{\partial t}-\dot{p}_{\alpha} v_{\alpha}-\dot{p}_{\alpha} x_{\alpha}=\frac{\partial \Pi}{\partial t}-\dot{E}-\sigma \dot{p}_{\alpha} x_{\alpha}
\end{gathered}
$$

в обоих вариантах. Следовательно,

$$
\dot{E}+\dot{\mathrm{II}}=\frac{\partial \Pi}{\partial t}-\sigma_{\alpha} x_{\alpha} .
$$

Ho

$$
\begin{gathered}
-\sigma p_{\alpha} x_{\alpha}=\sigma \frac{\partial \Pi}{\partial x_{\alpha}} x_{\alpha}=\sigma x_{\alpha} \frac{\partial \Pi}{\partial q_{\alpha}} e^{-\sigma t}=\frac{\partial \Pi}{\partial q_{\alpha}}\left(-\frac{\partial q_{\alpha}}{\partial t}\right)=-\frac{\partial \Pi}{\partial q_{\alpha}} \frac{\partial q_{\alpha}}{\partial t}= \\
=-\frac{\partial \Pi}{\partial t} .
\end{gathered}
$$

Поэтому в обоих вариантах

$$
E+\mathrm{II}=W=\text { постоянной. }
$$

$W$ означает здесь полную энергию, т. е. сумму из энергии собственной $E$ и энергии потенциальной П. Эта полная энергия при таком потенциале, стало быть, не зависит от эпохи $t$.

2) Ограничиваясь теперь вариантом $\mathrm{E}$, рассмотрим II, пропорциональное $M$, но в остальном зависящее лишь от $q_{\alpha}$, так что

Заметив, что здесь

$$
\Pi \equiv M \Phi\left(q_{1}, q_{2}, q_{3}\right) \text {. }
$$

$$
M=\frac{E}{c^{2}},
$$

можем записать этот потенциал и в виде

$$
\mathrm{II} \equiv \frac{E}{c^{2}} \Phi\left(q_{\alpha}\right)
$$

Тогда

$$
\dot{\mathrm{II}}=\frac{\partial \mathrm{II}}{\partial t}+\frac{\partial \mathrm{II}}{\partial x_{\alpha}} \dot{x}_{\alpha}+\frac{\dot{E}}{c^{2}} \Phi=\frac{\partial \mathrm{II}}{\partial t}-\dot{E}-\sigma \dot{p}_{\alpha} x_{\alpha}+\frac{\dot{E}}{c^{2}} \Phi .
$$

Но $E$ не содержит $t$ явно, вследствие чего попрежнему

$$
\frac{\partial \mathrm{II}}{\partial t}-\sigma \dot{p}_{\alpha} x_{\alpha}=0
$$

Следовательно,

$$
\dot{\mathrm{II}}=-\dot{E}+\frac{\dot{E}}{c^{2}} \Phi .
$$

С другой стороны,

$$
\dot{\mathrm{II}}=\frac{E}{c^{2}} \dot{\Phi}+\frac{\dot{E}}{c^{2}} \Phi,
$$

вследствие чего получается

$$
\dot{E}=-\frac{E}{c^{2}} \dot{\Phi},
$$


T. e.

$$
\frac{d E}{E}=-\frac{d \Phi}{c^{2}} .
$$

Понимая под $K$ некоторую постоянную, имеем на этом основании

$$
E=K e^{-\frac{\Phi}{c^{2}}}
$$

Пусть в какую-либо эпоху и в каком-то месте, занимаемом тогда частицей, $\Phi$ имело частное значение $\Phi_{1}$ и $E$ частное значение $E_{1}$. Находим

$$
\begin{aligned}
K & =E_{1} e^{\frac{\Phi_{1}}{c^{2}}} . \\
\text { Стало быть, полагая } \Phi-\Phi_{1} & =\Delta \Phi, \text { выводим } \\
E & =E_{1} e^{-\frac{\Delta \Phi}{c^{2}}} .
\end{aligned}
$$

Таким образом, собственная энергия здесь целиком определяется приростом $\triangle \Phi$. Это значит, что при одинаковых значениях $Ф$ и собственная энергия тоже должна иметь одинаковые значения в любые эпохи. Это выражение закона сохранения энергии в варианте Е.

В виду того, что

$$
E=m c^{2} \mathrm{ch \omega}, E_{1}=m c^{2} \mathrm{ch \omega}_{1} \text {, }
$$

можем записать (11) еще и в виде

$$
\operatorname{ch} \omega=e^{-\frac{\Delta \Phi}{c^{2}}} \operatorname{ch} \omega_{1},
$$

где $\omega$ и $\boldsymbol{\sigma}_{1}$ определяют собственные скорости в двух местах и эпохах при наличии разности $\Delta \Phi$ в не зависящем от $M$ множителе потенциала.

\section{4. Закон момента импульса}

В случае центрально-симметрического поля потенциал имеет вид

$$
\Pi \equiv \Pi\left(v_{\alpha}, \rho, t\right), \quad \rho^{2} \doteq x_{\alpha} x_{\alpha}=x_{1}{ }^{2}+x_{2}{ }^{2}+x_{3}{ }^{2} \text {. }
$$

Тогда вектор $p_{\alpha}$ всегда направлен через центр поля, прннятый нами в начале координат, и поэтому момент этого вектора относительно начала координат исчезает, т. е.

$$
x_{\alpha} \dot{p}_{\beta}-x_{\beta} \dot{p}_{\alpha}=0
$$

при любом сочетании двух индексов $\alpha, \beta$. В классической механике отсюда следует хорошо известным путем закон неизменности во времени момента самого импульса $p_{\alpha}$. В гиперболической механике такому заключению мешает слагаемое $\sigma \boldsymbol{x}_{\alpha}$ в правой части (1).

Нетрудно, однако, установить, что производная функции

$$
\mu_{\alpha \beta}=e^{-\sigma t}\left(x_{\alpha} p_{\beta}-x_{\beta} p_{\alpha}\right)
$$

исчезает тождественно. В самом деле,

$$
\begin{aligned}
& \dot{\mu}_{\alpha \beta}=e^{-\sigma t}\left[-\sigma\left(x_{\alpha} p_{\beta}-x_{\beta} p_{\alpha}\right)+\left(\dot{x}_{\alpha} p_{\beta}-\dot{x}_{\beta} p_{\alpha}\right)\right]= \\
& =e^{-\sigma t}\left[v_{\alpha} p_{\beta}-v_{\beta} p_{\alpha}\right]=e^{-\sigma t}\left[v_{\alpha} M v_{\beta}-v_{\beta} M v_{\alpha}\right]=0 .
\end{aligned}
$$


Это значит, что в гиперболической механике для любого центральносимметрического потенциала, функция $\mu_{\alpha \beta}$ неизменна во времени, а, стало быть, проекции момента импульса следуют закону

$$
x_{\alpha} p_{\beta}-x_{\beta} p_{\alpha}=\mu_{\alpha \beta} e^{\sigma t},
$$

где $\mu_{\alpha \beta}$ постоянные; другими словами, проекции момента импульса относительно центра поля в о р а с т а ют во времени по экспоненциальному закону. Эта особенность - результат влияния инерциальной скорости на движениіе частицы.

Из (12) следует, что траектория плоская, ибо вычисление дает

$$
\mu_{23} x_{1}+\gamma_{31} x_{2}+\mu_{13} x_{3}=0 \text {. }
$$

Перейдем к полярным координатам $\rho, \varphi$ в плоскости траектории, каковую всегда можно считать за плоскость $1-2$. Пусть $p_{p}, v_{p}$ соответстзєнно проекции импульса и собственной скорости на радиус-вектор, а $p^{q}$ $v_{\varphi}$ соответственно проекции на направление, перпендикулярное к радиус-вектору и считаемое положительным в сторону возрастающих $\varphi$. Тогда

$$
x_{1} p_{2}-x_{2} p_{1}=\rho p_{\varphi} .
$$

Вводя еще приведенное значение $q$ радиус-вектора $\rho$ посредством

получаем из

$$
q=p e^{-s t},
$$

$$
q p_{\varphi}=\mu_{12}=\text { постоянной. }
$$

Таким образом, классическая форма закона момента импульса справедлива при переходе к приведенному радиус-вектору.

Заметим, что из

$$
\dot{p} \dot{p}=x_{1} \dot{x}_{1}+x_{2} \dot{x}_{2}
$$

следует

то есть

$$
\rho=\frac{x_{1} v_{1}+x_{2} v_{2}}{\rho}+\sigma \rho=\frac{\rho v_{p}}{\rho}+\sigma \rho=v_{\rho}+\sigma \rho,
$$

$$
\dot{p}=v_{\rho}+\sigma \rho
$$

На этом основании легко получается, что

\section{Учитывая}

$$
\dot{q}=v p e^{-\sigma t} \text {. }
$$

находим

$$
x_{1}=\rho \cos \varphi, x_{2}=\rho \sin \varphi \text {, }
$$

$$
v_{1}+\sigma x_{1}=\dot{x}_{1}=\rho \cos \varphi-\rho \sin \varphi \cdot \dot{\varphi}=\left(v_{\rho}+\sigma \rho\right) \cos \varphi-x_{2} \dot{\varphi} r
$$

то есть

$$
v_{1}=v_{p} \cos \varphi-x_{2} \varphi .
$$

С другой стороны, по закону проекций,

$$
v_{1}=v_{\rho} \cos \varphi-v_{\varphi} \sin \varphi=v_{\rho} \cos \varphi-\frac{v_{\varphi} x_{2}}{\rho} .
$$


Из сопоставления двух значений, следовательно, получается:

$$
\dot{\varphi}=\frac{v_{\varphi}}{\rho}=\frac{p_{\varphi}}{M_{\varphi}},
$$

жак и в каждом из двух вариантов классической механики.

\section{5. Закон сохранения импульса системы}

В случае системы материальных точек, воздействующих друг на друга по ньютоновскому закону равенства действия и противодействия, но не подверженной влиянию внешних по отношению к системе воздействий, закон неизменности суммы импульсов сохраняется и в гиперболической механике. В самом деле, для такой системы сумма проекций векторов $\dot{p}_{\alpha}$ должна исчезнуть, вследствие чего

$$
\Sigma \dot{p}_{\alpha}=0, \quad \alpha=1,2,3
$$

Стало быть, сумма импульсов, т. е. $\Sigma p_{\alpha}$, представляет вектор, неизменный во времени.

Для варианта $\mathrm{N}$ отсюда и в гиперболической механике получается соответствующий закон движения центра тяжести системы, правда, с некото̄рой модификацией. Действительно, при

$M=m$, т. е. $\dot{M}=0$, имеем:

$$
\Sigma m v_{\alpha}=A_{\alpha}, \alpha=1,2,3 \text {, }
$$

тде $A_{\alpha}$ константы.

С другой стороны, для координат $\xi_{\alpha}$ центра тяжести

$$
\Sigma m x_{\alpha}=\xi_{\alpha} \Sigma m \text {, }
$$

T. e.

$$
\Sigma m \dot{x}_{\alpha}=\dot{\xi}_{\alpha} \Sigma m=\Sigma m\left(v_{\alpha}+\sigma x_{\alpha}\right)=\Sigma m v_{\alpha}+\sigma \Sigma m x_{\alpha} .
$$

Стало быть,

$$
\dot{\xi}_{\alpha} \Sigma m=A_{\alpha}+\sigma \xi_{\alpha} \Sigma m
$$

T.e.

$$
\dot{\xi}_{\alpha}=\frac{A_{\alpha}}{\Sigma m}+\sigma \xi_{\alpha} .
$$

Значит, центр тяжести движется с неизменной собственной скоростью $\frac{A_{\alpha}}{\Sigma m}$.

Обозначим компоненты этой неизменной собственной скорости посредством a . Решением дифференциального уравнения

$$
\dot{\xi}_{x}=a_{\alpha}+\sigma \xi_{\alpha}
$$

является

$$
a_{\alpha}+\sigma_{\alpha}=\left(\alpha_{\alpha}+\sigma_{\xi_{\alpha}}^{\circ}\right) e^{\sigma t},
$$

где $\xi_{\alpha}^{\circ}$ координаты центра тяжести в нулевую эпоху.

Исключая $e^{\sigma t}$ и полагая еще $\sigma^{-1}=\tau$, находим отсюда:

$$
\frac{\xi_{1}+\tau a_{1}}{\xi^{0}+\tau a_{1}}=\frac{\xi_{2}+\tau a_{2}}{\xi_{2}^{\circ}+\tau a_{2}}=\frac{\xi_{3}+\tau a_{3}}{\xi_{3}^{\circ}+\tau a_{3}}
$$


Это означает, что траектория центра тяжести прямолинейна и проходит через точку с координатами - $a_{\alpha}$; направляющие косинусы траектории пропорциональны величинам $\xi_{\alpha}^{3}+\tau a_{\alpha}$. В точке $-\tau a_{\alpha}$ центр тяжести находится в эпоху $t=-\infty$, что легко установить. Следовательно, траектория представляет пол у пр я м у ю, исходящую из названной точки. Возможен исключительный случай вырождения полупрямой именно в эту точку - $a_{\alpha}$; случай вырождения получается тогда, когда все знаменатели а, стало быть, и все числители, нули. Собственная скорость в этом и только в этом случае как раз компенсируется инерциальной скоростью, вследствие чего центр тяжести пребывает на одном месте.

Итак, при ньютоновском варианте гиперболической механики, центр тяжести изолированной системы должен описывать полупрямую и иметь неизменную собственную скорость; в исключительных случаях эта полупрямая может выродиться в одну точку.

Заметим, что в варианте $\mathrm{E}$ ньютоновское определение центра тяжести лишается смысла, ибо оно изменится прн переходе к другой инерциальной системе вследствие зависимости от понятия одновременности.

\section{6. Орбита планеты}

Вопрос о движении планеты в центрально-симметрическом поле тяготения решается сравнительно просто при варианте N. Вариант Е аналитически сложнее, но в первом приближении дает результаты, тождесгвенные с вариантом N; добавочные уточнения вносят поправки, относительная величина которых в случае солнечной системы не превышает порядка $10^{-7}$. Поэтому ограничимся здесь вариантом N.

По Ньютону потенциал центрально-симметрического поля тяготения имеет вид

$$
\Pi=-\frac{m \mu x^{2}}{\rho},
$$

где $m$ - неизменная масса планеты, $\mu$ - неизменная масса Солнца, $x^{2}=$ $6,68 \cdot 10^{-8}$ (в системе сантиметр-грамм-секунда) постоянная тяготения. Однако такой потенциал в гиперболической механике не удовлетворял бы закону сохранения полной энергии.

Эта неувязка устраняется, если вместо $x^{2}$ ввести $x^{2} e^{\text {ot }}$, чем вводится гипотеза о медленном вековом возрастании постоянной тяготения (в размере $1 \%$ в течение двух миллионов лет). Тогда получается потенциал консервативного типа, соответствующий случаю 1 в разделе 3 , а именно

$$
\Pi=-\frac{m \mu x^{2}}{q} .
$$

Для упрощения предполагается столь значительное преобладание $\mu$ над $m$, что движением Солнца относительно начала координат можно пренебречь, другими словами, взамен системы двух тел рассматриваем лишь одно тело, планету.

Закон сохранения полной энергии и закон момента импульса дают интегралы

$$
\begin{gathered}
\frac{1}{2} m v^{2}+\Pi=W \\
m q v_{\varphi}=S,
\end{gathered}
$$

где $W$ и $S$ постоянные. 
Кроме того, имеем

Следовательно,

$$
v_{\varphi}=\dot{\varphi}=\dot{\varphi} q e^{\sigma t}, v_{p}=\dot{q} e^{s t} .
$$

$$
\frac{v_{\varphi}}{v_{\rho}}=q \cdot \frac{\dot{\varphi}}{\dot{q}}=q \frac{d \varphi}{d q}, v^{2}=v_{p}{ }^{2}+v_{\varphi}{ }^{2} .
$$

Заметив, что (17), (18) и (19). отличаются от классических соотношений всего лишь заменой $\rho$ значением $q$, непосредственно заключаем, что зависимость между $q$ и $\varphi$ должна быть той же, что и между $\rho$ и $\varphi$ в классической проблеме. Достаточно поэтому лишь мимоходом отметить соответствующий путь решения.

Второе соотношение (18) дает

$$
v_{\varphi}=\frac{S}{m q},
$$

а первое означает

$$
m\left(v_{p}^{2}+v_{\varphi}{ }^{2}\right)=2(W-I I) .
$$

Поэтому

$$
1+\frac{u_{p}^{2}}{u_{\varphi}{ }^{2}}=\frac{2 m q^{2}(W-I I)}{S^{2}} .
$$

Учитывая еще значение II по (17), имеем поэтому

$$
\frac{u_{p}}{u_{\varphi}}=\frac{\sqrt{2 m W q^{2}+2 m^{2} \mu \times{ }^{2} q-S^{2}}}{S} .
$$

Годкоренное количество здесь представляет полином

$$
U(q)=A q^{2}+2 B q-S^{2},
$$

где

$$
A=2 m W, B=m^{2} \mu \kappa^{2} .
$$

Сличая это с (19), приходим к дифференциальному уравнению

$$
\frac{d q}{d \varphi}=\frac{1}{S} q \sqrt{U(q)}
$$

интегралом которого, при надлежащем выборе начального значения, является

$$
q=\frac{S^{2}}{B+\sqrt{B^{2}+A S^{2}} \cos \varphi},
$$

что легко проверяется непосредственной подстановкой. В процессе нахождения этого решения двузначность квадратного корня из $U(q)$ обходится введением униформизирующей переменной, каковой и оказывается само $\varphi$. В (20) квадратный корень из $B^{2}+A S^{2}$ подразумевается уже с неизменным положительным знаком.

(20) представляет в полярных координатах $q$, $\varphi$ коническое сечение с фокальной полухордой $P$,

$$
P=\frac{S^{2}}{B}=\frac{S^{2}}{m^{2} \mu x^{2}}
$$

и эксцентриситетом $s$,

$$
\varepsilon=\frac{\sqrt{B^{2}+A S^{2}}}{B}=\sqrt{1+\frac{2 W S^{2}}{m^{3} \mu^{2} \chi^{4}}} .
$$

При $W<0$ получается $s<1$, т. е. эллипс. 
Переходя к $\rho$, получаем истинную траекторию в виде

$$
\rho=\frac{P e^{\sigma t}}{1+\varepsilon \cos \varphi} \text {. }
$$

Это можно рассматривать, как разбухающее с течением времени коническое сечение.

Положим еще $\varphi=2 \pi$, тогда

$$
P=\frac{P e^{\sigma t}}{1+\varepsilon \cos 2 \pi} \text {. }
$$

Точки траектории, для которых > принимает целочисленные значения $n$, можно условно называть перигелиями, невзирая на то, что ввиду наличия множителя $e^{\text {бt } э т и ~ у с л о в н ы е ~ п е р и г е л и и ~ н е с к о л ь к о ~ о т л и ч а ю т с я ~ о т ~}$ мест минимальных $\rho$.

Переход от $n$ к $n+1$ будем называть одним оборотом планеты, а именно оборотом за номером $n+1$.

Для полного решения нужно еще определить зависимость $t$ от $\varphi$. Нас при этом в первую очередь интересует длительность $T_{n}$ оборота за номером $n$.

\section{7. Зависимость между временем и эксцентрической аномалией}

Из (16) и (18) получается

$$
\varphi q e^{\sigma t}=\frac{S}{m q},
$$

T. e.

$$
e^{-\sigma t} d t=\frac{m q^{2}}{S} d \varphi=\frac{m q d q}{\sqrt{U(q)}}
$$

Для удобства интегрирования правой части, здесь, как известно, полезно ввести эксцентрическую аномалию в качестве униформизирующей переменной, которую тут обозначим символом $\lambda$. Для этого полагаем

$$
q=\frac{S^{2}}{B+\sqrt{B^{2}+A S^{2}} \cos \varphi}=\frac{B-\sqrt{B^{2}+A S^{2}} \cos \lambda}{-A} .
$$

При $\cos \varphi= \pm 1$ это дает $\cos \lambda= \pm 1$, что легко установить. Поэтому, в частности, значению $\varphi=2 \pi n$ соответствует значение $\lambda=2 \pi n$.

При введении $\lambda$ получается

$$
U(q)=\frac{B^{2}+A S^{2}}{-A} \sin ^{2} \lambda, d q=\frac{\sqrt{B^{2}+A S^{2}}}{-A} \sin \lambda d \lambda,
$$

и найденное дифференциальное уравнение превращается в

$$
e^{-\sigma t} d t=\frac{m B}{(-A)^{3 / 2}}(1-s \cos i) d \lambda
$$

нбо

$$
\sqrt{B^{2}+A S^{2}}=B \varepsilon
$$

Интегрируя слева от 0 до $t$, а справа от 0 до $\lambda$, находим:

$$
-\left(1-e^{-\sigma t}\right)=\frac{m B}{(-A)^{3 / 2}}(\lambda-\varepsilon \sin \lambda) \text {. }
$$


Так как $\lambda$ как функция от чзадана, то тем самым из (24) получается и зависимость $t$ от $\varphi$. Вместе с тем и в (23) определяется зависимость $\rho$ от одного лишь

Имеем:

Полагая $\lambda=2 \pi n$, определяем из (24) соответствующие времена $t_{n}$

$$
=\left(1-e^{-\sigma t_{n}}\right)=2 \pi n \frac{m B}{(-A)^{3 / 2}} .
$$

Введем еще постоянную $T^{\circ}$ посредством

Тогда

$$
\frac{m B}{(-A)^{3 / 2}}=\frac{\mu m^{2 / 2} x^{2}}{2 \cdot \sqrt{2}(-W)^{3 / 2}}=\frac{T^{\circ}}{2 \pi} .
$$

$$
e^{-\sigma t_{n}}=1-\sigma n T^{\circ}
$$

Примем $n=1$ и развернем левую часть по степеням $\sigma t_{1}$. Получается

$$
\sigma t_{1}-\frac{1}{2} \sigma^{2} t_{1}^{2}+\frac{1}{6} \sigma^{3} t_{1}^{3}-\ldots=\sigma T^{\circ} \text {, }
$$

r e.

$$
t_{1}\left[1-\frac{1}{2} \sigma t_{1}+\frac{1}{6_{1}} \sigma^{2} t_{1}^{2}-\ldots .\right]=T^{\circ} .
$$

Для солнечной системы $\sigma t_{1}$ колеблется от $10^{-10}$ (Меркурий) до $10^{-7}$ (Плутон). Поэтому, положив

$$
t_{1}=T^{0}=\frac{\pi \mu m^{3 / 2} \kappa^{2}}{\sqrt{2}(-W)^{3 / 2}},
$$

мы внесем относительную погрешность, не превышающую $10^{-\tau}$. Эта погрешность далеко перекрывается неучетом разных добавочных обстоятельств в наших вычислениях, в частности неучетом возмущающего влияния остальных планет.

Бо́льшая точность, если считать наши выкладки строгими, все же получается, если положить

$$
t_{1}\left(1-\frac{1}{2} \sigma T^{\circ}\right)=T^{\circ},
$$

откуда

$$
t_{1}=\frac{T^{\circ}}{1-\frac{1}{2} \sigma T^{\circ}}
$$

что весьма близко к

$$
t_{1}=T^{\circ}\left(1+\frac{1}{2} \sigma T^{\circ}\right) .
$$

Подобным же методом определяем следующее приближение из

$$
t_{1}\left[1-\frac{1}{2} \sigma T^{\circ}\left(1+\frac{1}{2} \sigma T^{\circ}\right)+\frac{1}{6} \sigma^{2} T^{\circ 2}\right]=T^{\circ},
$$

что дает

$$
t_{1}=T^{\circ}\left(1+\frac{1}{2} \sigma T^{\circ}+\frac{1}{3} \sigma^{2} T^{\circ 2}\right)
$$

\section{8. Длительность периодов оборотов}

Очевидно,

$$
T_{1}=t_{1}, T_{n}=t_{n}-t_{n-1} .
$$


Заметим, что левая сторона в (25) не может быть отрнцательной, вследствие чего из

$$
1-\sigma n T^{\circ} \geq 0
$$

получаем неравенство

$$
n \leq \frac{\tau}{T^{\circ}}
$$

Положим еще

$$
\frac{\tau}{T^{\circ}}=N, \quad N T^{\circ}=\tau
$$

Определенное таким образом весьма большое число $N$ означает число остающихся еще на все будущее время оборотов планеты. Ограниченность этого числа оборотов объясняется увеличением их длительности. Для последнего оборота (или части его, если $N$ дробное) уже требуется бесконечно длительное время. Для Меркурия $N=8,1 \cdot 10^{\circ}$, для Плутона $N=$ $=7,9 \cdot 10^{6}$. Для Земли $N=2,0 \cdot 10^{9}$.

Перепишем теперь (25) в виде

$$
e^{-\sigma t} n=1-\frac{n}{N}
$$

отсюда

$$
e^{s t n}=\frac{N}{N-n}, e^{\sigma t_{n-1}}=\frac{N}{N-n+1}
$$

Следовательно,

$$
e^{\sigma\left(t_{n}-t_{n-1}\right)}=e^{\sigma T_{n}}=\frac{N-n+1}{N-n}
$$

T. e.

$$
e^{\sigma T_{n}}=1+\frac{1}{N-n}
$$

Поэтому

$\sigma T_{n}=\ln \left(1+\frac{1}{N-n}\right)=(N-n)^{-1}-\frac{1}{2}(N-n)^{-2}+\frac{1}{3}(N-n)^{-3}-\ldots$

H

$$
\sigma T_{1}=(N-1)^{-1}-\frac{1}{2}(N-1)^{-2}+\frac{1}{3}(N-1)^{-3}-\cdots
$$

Для большого $N$ и малых $n$ отсюда вытекает:

$$
\frac{T_{n}}{T_{1}} \approx \frac{N-1}{N-n^{\prime}}
$$

следовательно,

$$
T_{n}-T_{1} \approx \frac{n-1}{N} T_{1}
$$

В частности,

$$
T_{2}-T_{1}^{-}=\frac{1}{N} T_{1}
$$

Фбусловленное этим удлинение года для Земли составляет в секундах

$$
T_{2}-T_{1}=0,5 \cdot 10^{-9} \cdot 3,16 \cdot 10^{7}=1,6 \cdot 10^{-2} \text {. }
$$


Подобное же вычисление для Меркурия дает

а для Плутона

$$
\therefore T_{2}-T_{1}=9,4 \cdot 10^{-4} \text {, }
$$

$$
T_{2}-T_{1}=990 .
$$

Отметим еще примерные величины годичного увеличения расстояний от Солнца. Так как

$$
\rho_{1}=\rho_{0} e^{\sigma t_{1}}=\rho_{0} \frac{N}{N-1} \approx \rho_{0}\left(1+\frac{1}{N}\right),
$$

где $\rho_{\circ}$ и $\rho_{1}$ последовательные расстояния в перигелии, то приращение этого расстояния составляет за один оборот $\frac{1}{N}$ P.

означает приращение в 6 метров за каждый современный оборот, для Земли годичное приращение в 75 метров, а для Плутона в 600 километров за один современный оборот.

Все названные эффекты по своей малости вряд ли поддаются непосредственному наблюдению. Удлинение земного года к тому же, вероятно, затушевывается эффектом удлинения секунды вследствие тормозящего влияния приливной волны на вращение земного шара.

\section{9. Возрасты планет солнечной системы}

Гиперболическая механика дает новый подход к вопросам космогонии солнечной системы на основании представления об отделении планет от вращающегося центрального тела - Солнца.

Если сгусток вещества, образовавший планету, отделился от Солнца в эпоху $t$ под влиянием центробежной силы, то при соответствующем радиусе $r$ и угловой скорости w вращения Солнца в ту эпоху должно было иметь место соотношение

$$
w^{2} r=\frac{\mu \times^{2} e^{\sigma t}}{r^{2}}
$$

Это соотношение выражает равенство между величиной центробежной силы и величиной ньютоновского притяжения на периферии, т. е. выражает то, что в такую эпоху соответствующие частицы вещества описывали круговые траектории уже не в силу сцепления вещества Солнца, а под влиянием поля тяжести.

С момента отделения планета, в основном, должна подчиняться уже законам планетного движения. Но из (23а) тогда явствует, что

$$
r=p_{0} e^{\sigma t} \text {, }
$$

если $\rho_{\circ}$ расстояние перигелия планеты от Солнца на сегодняшний день. Поэтому требование (30) означает

$$
w=\sqrt{\frac{\mu x^{2}}{\rho_{0}^{3}}} e^{-\tau t}
$$

Если бы был известен закон изменения w во времени, причем на сегодня $w$ имеет значение $w_{\circ}=2,882 \cdot 10^{-6}\left[\mathrm{cek}^{-1}\right]$, то из (30а) можно было бы определить $t$ а затем уже и $r$. Четких данных для установления закона изменения w нет; вероятно лишь то, что ж' возрастало с течением времени, хотя бы под влиянием сокращения размеров Солнца. 


$$
w=w_{0} e^{o t},
$$

для планет получаются результаты, хорошо согласующиеся с оценками возраста Земли и метеоритов другими методами, а именно, на основании изучения радиоактивности соответствующего вещества.

При гипотезе (31) соотношение (30а) дает:

$$
e^{2 \sigma t}=\frac{1}{w_{0}} \sqrt{\frac{\mu \kappa^{2}}{p_{0}^{3}}} .
$$

Для более удобного вычисления отсюда величины $t$ можно поступить следующим образом.

Взяв десятичный логарифм, получим из (32)

$$
t=\frac{-\log w_{0}+\frac{1}{2} \log \mu x^{2}-\frac{3}{2} \log \rho_{0}}{2 \cdot 1,6 \cdot 10^{-17} \cdot 0,4343}
$$

в секундах. Чтобы получить $t$ в миллиардах земных лет, надлежит разделить это количество еще на

$$
10^{9} \cdot 365,26 \cdot 86400=3,16 \cdot 10^{16} .
$$

Таким образом, в миллиардах лет,

$$
t=2,28\left[-\log w_{\circ}+\frac{1}{2} \log \mu x^{2}-\frac{3}{2} \log p_{0}\right] .
$$

Здесь $\rho_{\text {o }}$ еще выражено в сантиметрах. Переходя к миллионам километров, нам надлежит увеличить $\log \rho_{\text {o }}$ на 11 единиц. Вводя еще численные значения $\log w_{\circ}$ и $\log \mu x^{2}$, получаем в новых единицах

$$
t=2,28\left[5,5403+13,0624-\frac{3}{2}\left(11+\log \rho_{0}\right)\right]
$$

что в конечном итоге приводит к удобной для табулирования формуле

$$
t=4,79-3,42 \log p_{\circ},
$$

где $\rho_{\circ}$ вводится в миллионах километрах, a $t$ получается в миллиардах лет. Для солнечной системы (33) дает отрицательные $t$, как и должно быть, ибо отделение планет должно было происходить в прошлом.

Зная $t$, можно определить уже и $r$ по формуле

$$
r=p_{0} e^{0,5 t} \text {. }
$$

В самом деле, если $t$ дано в миллиардах лет, то $\sigma$ соответственно умножается на $3,16 \cdot 10^{16}$; но

$$
1,6 \cdot 10^{-17} \cdot 3,16 \cdot 10^{16}=0,505,
$$

чем и подтверждается пригодность формулы (34).

\section{0. Космогонические соображения}

Результаты применения формул (33) и (34) к планетам солнечной системы даны в нижеследующей таблице, где Церера включена как представитель группы малых планет.

По данным радиоактивности, возраст Земли оценивается в промежутке от 2 до 3 миллиардов лет; таблица дает 2,6. Возрасты метеоритов, по данным радиоактивности, покрывают широкую шкалу, причем возраст наиболее молодых метеоритов определяется несколькими сотнями миллионов лет, а наиболее старых от 7 до 8 миллиардов лет. По данным таблицы для наиболее молодой планеты получается возраст в 900 миллионов лет, а для наиболее старых - возраст в 7,7 миллиардов лет.Если считать, что 
метеориты родственны планетам, т. е. по существу являются частями самой солнечной системы, то и такое совпадение амплитуды возрастов следует признать вполне приемлемым.

Таблица

\begin{tabular}{|c|c|c|c|c|}
\hline Планета & $\left(10^{9}\right.$ лет) & $\begin{array}{c}r \\
\left(10^{6} \mathrm{KM}\right)\end{array}$ & $\begin{array}{c}w \\
\left(10^{-6} \text { ceк }^{-1}\right)\end{array}$ & $\begin{array}{l}3 \frac{\Delta r}{r} \\
(0 / 0)\end{array}$ \\
\hline 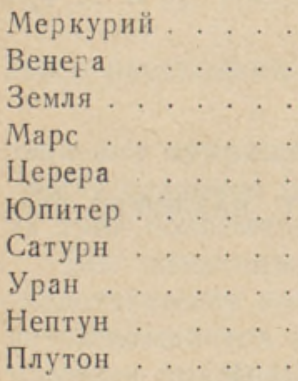 & $\begin{array}{l}-0,89 \\
-\quad 2,2 \\
-2,6 \\
-3,1 \\
-4,0 \\
-5,0 \\
-5,9 \\
-7,0 \\
-7,7 \\
-7,7\end{array}$ & $\begin{array}{l}29,4 \\
36,6 \\
39,2 \\
43,0 \\
49,5 \\
58,5 \\
68,5 \\
81,8 \\
92,2 \\
92,2\end{array}$ & $\begin{array}{l}1,84 \\
0,98 \\
0,77 \\
0,60 \\
0,37 \\
0,23 \\
0,15 \\
0,09 \\
0,06 \\
0,06\end{array}$ & $\begin{array}{l}0,04 \\
0,2 \\
0,2 \\
0,07 \\
0 \\
4,0 \\
2,0 \\
0,8 \\
0,9\end{array}$ \\
\hline
\end{tabular}

Малый эксцентриситет планетных орбит говорит в пользу первоначального участия соответствующей массы в кругообразном вращении экваториальной части периферии Солнца. Большие эксцентриситеты орбит метеоритов наводят на мысль, что метеориты образованы не вследствие центробежной силы, а, если они солнечного происхождения, образованы благодаря эруптивному выделению небольших количеств вещества из Солнца В таком случае можно ожидать, что плоскости их орбит расположены боллее или менее случайно; наибольший шанс встретиться с Землей, разумеется, имеют те метеориты, плоскость орбиты коих близка к плоскостн эКлиптики.

Небольшие отклонения плоскостей орбит планет можно объяснить шатанием оси вращения Солнца, вероятным при каждом отделении значительной планетной массы.

Отметим еще следующие соображения.

Из (30а) следует, что радиус Солнца сокращался примерно по закону

$$
r=\sqrt[3]{\frac{\mu x^{2}}{w_{0}^{2}}} e^{-1 / s \sigma t}
$$

Однако при таком законе и прочих прежних предпосылках на экваторе Солнца должно было бы неизменно господствовать состояние неустойчивости, что мало вероятно. Поэтому отделение планеты надлежит рассматривать как скачок Солнца от состояния неустойчивого к состоянию временно более устойчивому. При такой концепции имеют смысл нижеследующие ориентировочные подсчеты.

Вероятно плотность Солнца в любую эпоху возрастала к центру. Хотя закон этого возрастания и неизвестен, но в первом приближении можно считать этот закон линейным, характеризуя периферию как то место, где плотность нулевая. Считая притом Солнце за сферу, из этих условий определяем коэффициенты линейного закона распределения плотности

$$
\gamma=-a x+b
$$


где $b=a r$, ибо $\gamma=0$ при $x=r$. Так как общая масса Солнца равна $\mu$, то

$$
\int_{0}^{r} 4 \pi x^{2}(-a x+a r) d x=\mu .
$$

Отсюда легко находится, что

$$
a=\frac{3 \mu}{\pi r^{4}} .
$$

Периферийный слой толшины $\Delta r$ имеет при этом массу

$$
\int_{r-\Delta r}^{r} 4 \pi x^{2} \frac{3 \mu}{\pi r^{4}}(r-x) d x
$$

что с точностью второго порядка для малой величины $\Delta r$ равно

$$
6\left(\frac{\Delta r}{r}\right)^{2} \mu
$$

Но при таком предположении допустимо считать

$$
6\left(\frac{\Delta r}{r}\right)^{2} \mu=m
$$

если $m$ - масса только что отделившейся планеты. Принимая за единицу массу Земли, имеем $\mu=0,33 \cdot 10^{6}$. Поэтому получаем формулу

$$
\frac{\Delta r}{r}=\sqrt{\frac{m}{2}} \cdot 10^{-3}
$$

Быстрое сокращение радиуса на $\Delta r$ при еще не успевшем измениться ш дает относительное снижение центробежной силы на величину $\frac{\Delta r}{r}$, что легко уразуметь. Но, с другой стороны, снижение радиуса на величну $\Delta r$ повлечет за собой еще и увеличение силы тяготения в районе периферии. Так как величина этой силы

$$
\frac{m \mu x^{2}}{r^{2}}
$$

то изменению $-\Delta r$ соответствует увеличение на

$$
2 \frac{m \mu x^{2}}{r^{3}} \triangle r
$$

Поэтому относительное увеличение силы тяготения составляет $2 \frac{\Delta r}{r}$.

В итоге, относительное изменение соотношения центробежной силы и силы тяготения, бывших до отделения планеты в равновесии, составляет $3 \frac{\Delta r}{r}$

Эта величина и дана в четвертом столбце таблицы в процентах, с учетом масс соответствующих планет.

Значение $3 \frac{\Delta r}{r}$ в таком толковании измеряет запас временной устойчивости вещества периферии Солнца после только что свершившегося отделения планеты. По мере дальнейшего нарастания w этот запас устойчивости расходутся, если попутное уменьшение $r$ не идет достаточно быстро. Поэтому некоторое время спустя могут создаться условия для рождения новой планеты и т. д. Процесс прекратится, когда по какой-либо при- 
чине сокращение $r$ окажется довлеющим, увеличивая запас устойчивости при нарастании времени. Заметим попутно, что и вековое нарастание постоянной тяготения представляет фактор, влияющий в направлении увеличения запаса устойчивости. Поэтому промежутки времени между эпохами рождения двух последовательных планет могут быть достаточно зна чительными, если отделившаяся масса не слишком мала.

После рождения Меркурия сокращение Солнца пошло восьма ускоренными темпами: примерно за 1 миллиард лет $r$ сократилось от 30 миллионов километров до 0,7 миллионов, между тем как за предшествующие 6,5 миллиардов лет сокращение радиуса составило 60 миллионов километров, т. е. шло в 3 раза медленнее. Если так, то послемеркуриальный период характеризуется быстрым повышением температуры Солнца, что, быть может, каким-то образом увязано с установлением углеродного цикла перехода водорода в гелий.

После отделения Юпитера возник относительно большой запас устойчивости - 4\%. Все же близость массивного Юпитера могла обусловить на Солнце приливные бугры, в которых небольшая часть массы выходила из сферы устойчивости. Әто могло вызвать многократное отделение сравнительно малых планет за весь промежуток времени от Юпитера до Марса. Отделение Марса в таком случае определяет завершение этой неспокойной полосы времени: с одной стороны, получился новый, хотя и небольшой запас устойчивости; с другой стороны, Юпитер удалился на более значительное расстояние, так что его возмущающее действие перестало играть заметную роль. Во всяком случае, от Юпитера до Марса прошел исключительно большой промежуток времени, на протяжении которого вопросы устойчивости находили компромиссные решения путем выделения большого числа мелких планет без прямого влияния на запас устойчивости, который был для недеформированной поверхности Солнца достаточным.

Отделение Плутона и Нептуна произошло, повидимому, в одну и ту же эпоху, притом Плутон, должно быть, отделился несколько раньше, так как он подходит к Солнцу (в настоящее время) на 18 миллионов километров ближе, нежели Нептун. Возможно, что рождением двойни объясняются некоторые особенности у Нептуна и Плутона.

Вообще отделению планеты, вероятно, предшествовало образование грушевидного бугра на Солнце. Отделение кольца неправдоподобно, ибо это потребовало бы весьма невероятного стечения обстоятельств идеально равномерного распределения неустойчивости по солнечному экватору. Вращение почти всех планет вокруг своей оси в ту же сторону, как и Солнца, можно объяснить разностью скоростей у основания и у шейки грушевидного бугра.

Одним из серьезнейших возражений против восходящих к Канту и Лапласу «гипотез вращения» в космогонических теориях развития солнечной системы является то, что планеты, содержащие суммарно только 1/800 массы Солнца, все же захватили около $98 \%$ суммарного момента импульса всей солнечной системы. С классической механикой это не увязывается. Однако гиперболическая механика значительно ослабляет это веское возражение: по формулам гиперболической механики суммарный момент импульса планет за время их самостоятельного существования должен был увеличиться в 25 раз. Если наряду с этим допустить еще значительное уменьшение момента импульса самого Солнца вследствие сокращения с резким повышением температуры в послемеркуриальный период, другими словами, вследствие сокращения, не компенсированного соответствующим нарастанием угловой скорости вращения, то переход суммарного момента импульса солнечной системы к гланетам в основном становится понятным. 
Гиперболическая механика, формально сводящаяся к модификации закона инерции, исходя из наблюдений спектров внегалактических туманностей, математически отображается перемещением трехмерной предельной сферы в четырехмерном пространстве Лобачевского.

Соотношения, вытекающие из концепций гиперболической механики, подтверждают наличие борьбы противоположно устремленных процессов собирания и распыления в природе. Эта борьба осложняется еще непрестанными переходами форм материи и переходами в излучение. В этом ярко проявляется диалектика природы. 